\title{
Implantação de Serviços de Engenharia Clínica no Hurnp/Uel
}

\section{Clinical Engeneering Experience at the Hospital of the State University of Londrina}

\author{
Ernesto Fernando Ferreyra Ramírez ${ }^{1}$
}

\begin{abstract}
Resumo
Neste trabalho é relatada a experiência de implantação de serviços de engenharia clínica no Hospital Universitário Regional do Norte do Paraná (HURNP/UEL), via projeto de extensão do Departamento de Engenharia Elétrica da UEL. Neste projeto, buscou-se a melhoria da qualidade do parque tecnológico instalado, por meio da resolução de problemas no gerenciamento e manutenção dos equipamentos médicos e de seus sistemas de suporte. Com isso, pretende-se começar a formação de recursos humanos na área de Engenharia Clínica na região de Londrina, contribuindo para a melhoria do atendimento à população abrangida pelo HURNP, por meio da melhoria das condições de trabalho, aprendizado e ensino dos profissionais da área de saúde (docentes, alunos e funcionários) que utilizam equipamentos médicos nos serviços prestados à comunidade.
\end{abstract}

Palavras-Chave: Engenharia Clínica, Equipamentos médicos, Gerenciamento de Manutenção

\begin{abstract}
This paper describes the four-year experience of implementation of Clinical Engineering services at the Hospital of the State University of Londrina (HURNP/UEL). It was performed by the Electrical Engineering Department (DEEL), through a project involving lecturers and students from the Electrical and Civil Engineering Courses of the same university. The main objectives were the formation of human resources in the Clinical Engineering area and a positive contribution to the healthcare services offered by the HURNP for the community in the surroundings of Londrina - Paraná State - Brazil.

Key words: Clinical Engineering; Medical Equipment; Maintenance Management.
\end{abstract}

\section{Introdução}

Nos últimos 100 anos, a dependência do sistema de saúde em relação à tecnologia médica para a realização de serviços tem crescido continuamente. Até certo ponto, todos os profissionais das áreas de saúde dependem da tecnologia, seja na área de medicina preventiva, diagnóstico, cuidados terapêuticos, reabilitação, administração, seja no treinamento e educação relacionados à saúde. Assim, a tecnologia médica permite aos médicos o atendimento aos seus pacientes de maneira eficiente, segura e com um baixo custo em relação aos serviços prestados (BRONZINO 1992; ENDERLE et al., 2000). Entretanto, esta evolução não foi acompanhada pelas suas respectivas metodologias de controle (HAWKINS, 1992). Isso mostrou a utilidade de estabelecer departamentos de engenharia clínica nos hospitais, para cuidar dos aspectos técnicos e administrativos en-

\footnotetext{
${ }^{1}$ Prof. M.Sc. do Departamento de Engenharia Elétrica, Centro de Tecnologia e Urbanismo-UEL. Email:ernesto@uel.br.
} 
volvendo a operação segura e eficiente dos equipamentos médicos (PANOUSIS et al., 1997). Por isso, desde a década de 70, a atuação do engenheiro clínico, o qual possui formação básica em engenharia e conhece técnicas de gerenciamento de tecnologias médicas, vem ganhando realce nos hospitais do Brasil e do mundo (RAMÍREZ; CALIL, 2000). Sendo a engenharia clínica o setor responsável por todo o ciclo de vida da tecnologia, este setor deve participar do processo de aquisição, recebimento, testes de aceitação, treinamento, manutenção, alienação e demais assuntos referentes aos equipamentos médicohospitalares (ANTUNES et al., 2002).

A engenharia clínica possibilita a redução de custos e aumenta a eficácia dos procedimentos relacionados com a tecnologia na saúde. Segundo Betts (1987), as maiores reduções de custos ocorrem nas áreas de manutenção e aquisição de capital, mas economias adicionais são possíveis por meio do treinamento dos usuários ou pessoal de manutenção, gerenciamento de riscos, investigação de acidentes, e construção ou reforma do espaço físico. Para isso, o engenheiro clínico precisará coletar dados pertinentes e confiáveis que identifiquem as áreas que apresentam potencial para redução de custos.

Assim, podem-se citar algumas atribuições do engenheiro clínico em um hospital:

\section{$\underline{\text { Apoio Científico destinado a }}$}

- realizar pesquisas para desenvolvimento de instrumentação biomédica;

- sugerir adaptações e melhorias em equipamentos médicos;

- avaliar a relação custo-benefício de tecnologias médicas.

\section{Apoio Técnico para:}

- acompanhar o ciclo de vida dos equipamentos médicos (instalação, operação, manutenção corretiva, manutenção preventiva e desativação) para garantir o melhor nível possível de segurança dos seus usuários e pacientes;
- acompanhar e auxiliar os setores clínicos nos processos de licitação de equipamentos médicos, fornecendo especificações técnicas dos equipamentos desejados;

- fornecer treinamento, interno e externo, aos usuários e aos técnicos de manutenção dos equipamentos médicos.

\section{$\underline{\text { Apoio Gerencial destinado a: }}$}

- auxiliar na gerência de contratos de manutenção externa de equipamentos médicos;

- participar na padronização de procedimentos administrativos de solicitação de reparos, manutenções de rotina, emergências e manutenções preventivas de equipamentos médicos;

- otimizar custos durante a vida útil dos equipamentos médicos (instalação, operação, manutenção corretiva, manutenção preventiva e desativação).

Apesar de estar difundida no exterior, principalmente nos Estados Unidos, desde o início da década de 70, a engenharia clínica no Brasil ainda está na fase de criação de recursos humanos com a existência de cursos, desde 1993, que inicialmente foram financiados pelo Ministério da Saúde para formar pessoas nesta área (RAMÍREZ; CALIL, 2000). Devido às crescentes pressões governamentais (por meio de portarias da Vigilância Sanitária) e da sociedade civil (mediante a exigência por melhores serviços com custo cada vez mais baixo por parte das empresas em geral), a necessidade de profissionais nesta área é crescente. Isso se deve à grande demanda por serviços de assessoria técnica na parte de gerenciamento de tecnologias, principalmente nas instituições públicas de saúde, nas quais fazem muitas compras impróprias devido à falta de orientação dos setores de compras e à lei das licitações (LOPES 1993; CALIL 1994; GOMES; GOMES 1998; ESPERANÇA et al., 1998; SOUZA et al., 2000). Além disso, ainda não existe uma orientação adequada quanto à busca da melhoria da segurança e do desempenho dos equipamentos médico-hospitalares ao longo da sua vida útil, por meio da implementação 
de programas de treinamento aos usuários e de manutenção preventiva nestes equipamentos. Observase que nem sempre as etapas básicas num processo de desenvolvimento e gerência estão sendo articuladas entre o planejamento, o projeto, a execução, a manutenção e a operação. Geralmente, a preocupação maior é concentrada na execução de obras e na compra de equipamentos; a manutenção e operação, até por uma questão cultural, estão sendo negligenciadas, ocorrendo descontinuidades e baixa qualidade na prestação de serviços e aquisições (CORNIALI; LEITE, 2002). Isso é o que gera atrasos nas compras de equipamentos médicos e obriga o Brasil a pagar juros sobre dívidas, feitas junto a bancos estrangeiros, para aquisição de equipamentos, sem que a população consiga usufruir dos benefícios decorrentes destas (SOUZA et al., 2000). Para ter-se uma idéia do que isso representa de prejuízo ao Brasil, podem-se estimar gastos da ordem de US\$ 71 milhões anuais com mão-de-obra de manutenção de equipamentos (CALIL, 2000), os quais, junto com os gastos com peças e importação de equipamentos de imagens médicas (da ordem de US\$ 1,19 bilhão entre 1994 e 1998), representaram importantes fontes de evasão de divisas brasileiras em um mercado nacional estimado em US\$ 1,3 bilhão por ano (CALIL, 2001).

Essa realidade não diferia muito da encontrada no final da década de 80 por Netto et al. (1989), quando foi realizada uma pesquisa de diagnóstico dos serviços de Engenharia Hospitalar no Estado do Paraná, através do Centro Federal de Educação Tecnológica (CEFET-PR). Dentre os principais resultados obtidos salientavam-se: inexistência de setor específico para manutenção de equipamentos; infra-estrutura deficiente; carência de programas de segurança elétrica de equipamentos com relação a pacientes e operadores; e interesse na implantação de equipes próprias e na contratação de um profissional técnico qualificado na área (no caso um engenheiro clínico).

Em 1998, apesar de terem-se passado quase 10 anos, situação parecida foi encontrada no Hospital
Universitário Regional do Norte do Paraná (HURNP/UEL), quando da implantação de serviços de engenharia clínica nesse hospital, por meio de Departamento de Engenharia Elétrica (DEEL) da Universidade Estadual de Londrina (UEL). Assim, neste artigo será mostrada essa experiência de implantação, incluindo: os problemas encontrados, os procedimentos adotados e os resultados obtidos em 4 anos de trabalho.

\section{Histórico do Projeto}

A Universidade Estadual de Londrina (UEL) é uma universidade pública de 30 anos, que oferece mais de 100 cursos entre graduação, especialização, mestrado e doutorado, para um total de 15.000 alunos. Nela são realizados em torno de 500 projetos de pesquisa, ensino e extensão. Entre os cursos oferecidos pela UEL, existe o curso de Engenharia Elétrica, que foi criado em 1997, e atualmente é ofertado pelo Departamento de Engenharia Elétrica (DEEL/ CTU/UEL). Atualmente, o DEEL conta com 18 professores (55\% doutores, 39\% doutorandos e $4 \%$ mestres) para atender mais de 200 alunos de graduação e mestrado em diversas áreas de concentração. Entre elas, existe a área de "Instrumentação Biomédica, Eletrônica, Sensores, Semicondutores e Processamento da Informação", da qual o autor deste presente trabalho faz parte.

A UEL também possui um hospital-escola, o Hospital Universitário Regional do Norte do Paraná (HURNP/UEL), de 289 leitos, todos disponíveis ao SUS, e onde trabalham 1.750 servidores e 350 docentes, e estudam mais de 1.000 alunos de graduação e pós-graduação. O HURNP atende a todas as especialidades médicas, e efetua mensalmente 1.150 internações, 12.000 atendimentos ambulatoriais, 7.000 atendimentos em Pronto Socorro e 600 cirurgias. No HURNP, o parque tecnológico é composto por mais de 20.000 itens (em torno de 180 tipos de equipamentos), possuindo equipamentos de alta tecnologia (Tomografia Computadorizada, Ultra-som com Doppler colorido, Litotripsia Extracorpórea e 
Hemodinâmica). Além disso, nos últimos anos tem havido uma maior preocupação da Direção do hospital com a melhoria da qualidade dos serviços oferecidos. Por isso, o HURNP já vem participando em diversos programas do Ministério da Saúde, tais como: Rede de Hospitais Sentinela da Agência Nacional de Vigilância Sanitária (ANVISA), Centros de Colaboradores em Gestão Hospitalar, Humanização da Assistência Hospitalar e Modernização Gerencial de Grandes Estabelecimentos de Saúde. Dentro desta nova perspectiva, a Diretoria do HURNP requisitou o auxílio do autor deste trabalho, para o desenvolvimento de atividades relacionadas à gestão de tecnologias biomédicas, ou seja, em engenharia clínica no HURNP.

Assim, durante o período de 09/09/1998 a 06/04/ 1999, foi realizado um trabalho de diagnóstico dos problemas que estavam ocorrendo com os equipamentos médicos do HURNP. Para isso foram feitos os seguintes procedimentos:

- levantamento do parque tecnológico do hospital;

- acompanhamento das rotinas de manutenção corretiva realizadas no hospital;
- acompanhamento dos processos administrativos associados ao ciclo de vida (aquisição, manutenção e desativação) dos equipamentos médicos;

- implementação de alguns procedimentos de manutenção preventiva e de rotina em alguns equipamentos;

- verificação e negociação dos contratos de serviços de manutenção externa dos equipamentos;

- conscientização do pessoal das áreas de saúde do hospital sobre alguns aspectos de segurança elétrica;

- participação nos projetos de novas centrais de sistemas de apoio para o hospital.

A partir desses procedimentos, montou-se um Diagrama de Ishikawa (também conhecido como diagrama de causa-efeito ou espinha-de-peixe) mostrando os problemas encontrados no hospital relacionados ao parque de equipamentos do HURNP (Figura 1). Com base nesse diagrama, foram feitas as seguintes propostas à Direção do hospital para diminuir os problemas com equipamentos:

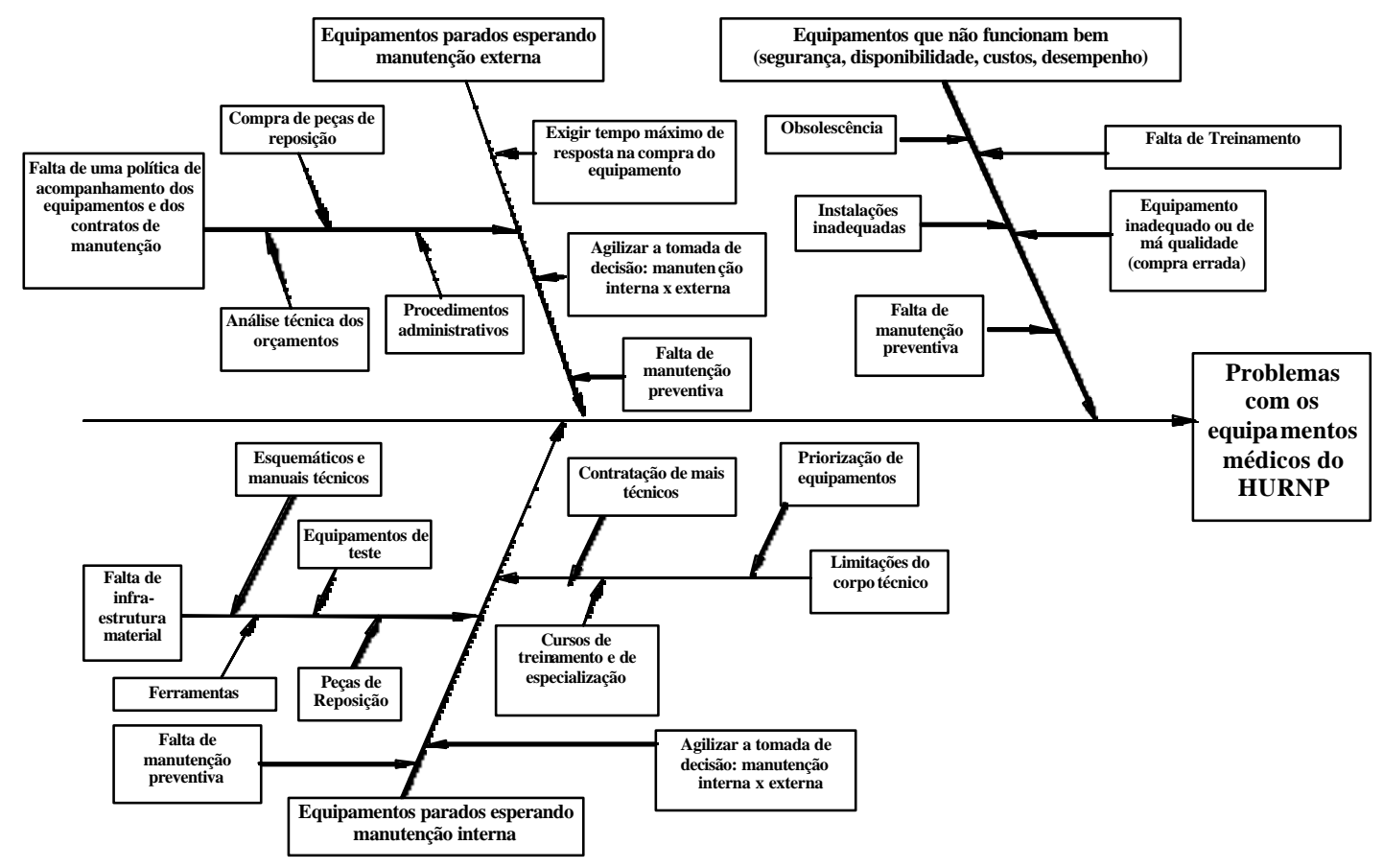

Figura 1. Principais problemas encontrados no HURNP relacionados ao parque de equipamentos. 
Investimentos na manutenção interna do hospital através da:

- aquisição de novas ferramentas;

- compra de equipamentos de teste para calibrar e testar os equipamentos após a manutenção;

- aquisição de esquemáticos e manuais de manutenção e de operação dos equipamentos;

- agilização dos procedimentos de compra de peças de reposição;

- viabilização de cursos internos/externos de treinamento e de qualificação do corpo técnico;

- contratação de mais técnicos ou de ajudantes.

Mudancas em alguns procedimentos nos processos de aquisição e manutenção de novos equipamentos por meio da:

- verificação, junto ao futuro usuário do equipamento, do que se quer comprar exatamente, fazendo uma verificação técnica e clínica para estudar a viabilidade e utilidade da aquisição para o hospital;

- exigência, junto aos vendedores dos equipamentos, de um tempo máximo de resposta (a ser definido pelo setor requisitante do equipamento) em caso de quebra ou falha do mesmo durante o período de garantia;

- exigência junto aos vendedores dos equipamentos, treinamentos técnicos e clínicos com fornecimento de manuais e esquemáticos, quando for o caso, estabelecendo parcerias para agilizar e baratear a manutenção dos equipamentos;

- liberação do pagamento dos equipamentos quando estes já estiverem instalados e funcionando regularmente;

- agilização dos procedimentos para fazer as compras de peças de reposição e pagar os serviços de urgência, podendo estes procedimentos ser atrelados a uma metodologia de priorização de equipamentos;
- agilização da obtenção de informações sobre os equipamentos médicos para fazer as licitações.

Avaliação da viabilidade da manutenção interna ou da terceirização da manutenção para cada tipo de equipamento médico do hospital através do(a):

- levantamento das condições de cada equipamento;

- determinação e estimativa dos custos de conserto (Hora técnica e tempo de conserto);

- avaliação da importância estratégica do equipamento (grau de utilização, número de equipamentos de reserva);

- implementação de um cadastro dos prestadores de serviços externos (Quem são? Qual o tipo de serviço que realizam? Quanto tempo levam para atender? Quanto custam os serviços?);

- criação de índices/parâmetros de comparação das alternativas disponíveis (por exemplo: tempo de resposta/atendimento; tempo médio de conserto; custo/qualidade do serviço; prioridade do equipamento para o hospital; etc.).

Implementação de um Programa de Manutenção Preventiva dos equipamentos médicos do hospital através da(o):

- avaliação da viabilidade e da necessidade de implementação de manutenção preventiva em cada um dos equipamentos do hospital;

- investimentos em recursos materiais e humanos no caso de ser mais viável, ou necessário, realizar a manutenção preventiva dos equipamentos médicos do hospital através do corpo de técnicos do HURNP;

- estabelecimento de parcerias com terceiros ou com os fornecedores dos equipamentos, realizando contratos de manutenção preventiva quando não for viável a participação do corpo de técnicos do HURNP. 
Verificacãa do estado real dos equipamentos médicos do hospital:

- identificação e fornecimento de laudos de desativação nos equipamentos que estavam realmente obsoletos e estudo de alternativas para fazer a sua substituição.

Visto que era impraticável a implementação simultânea de todas estas sugestões, devido às limitações de recursos materiais e humanos qualificados encontrados no HURNP e na própria UEL, optou-se por realizar um projeto de extensão envolvendo docente e alunos cursos de Engenharia da UEL. Estes deveriam estar dispostos a resolver alguns dos problemas do HURNP, e, com isso, poderiam ganhar experiência e servir à comunidade externa e interna da Universidade. A partir daí, inicialmente foi proposta a resolução de 04 (quatro) problemas mais imediatos do hospital:

- minimização dos efeitos do "bug do milênio" no parque de equipamentos do HURNP;

- assessoria técnica e avaliação tecnológica de equipamentos que estão sendo adquiridos pelo HURNP por meio de um convênio com o Ministério da Saúde (REFORSUS);

- implementação de um programa de manutenção preventiva nos equipamentos do HURNP;

- redimensionamento dos sistemas de gases, principalmente de ar comprimido do HURNP.

Assim, este projeto buscou a melhoria da qualidade do parque tecnológico instalado no Hospital Universitário Regional do Norte do Paraná (HURNP), por meio da resolução de problemas no gerenciamento e manutenção dos equipamentos médicos e seus sistemas de suporte. Com isso, pretende-se começar a formação de recursos humanos na área de Engenharia Clínica na região de Londrina, contribuindo-se para a melhoria do atendimento à população abrangida pelo HURNP, por intermédio da melhoria das condições de trabalho, aprendizado e ensino dos profissionais da área de saúde (docentes, alunos e funcionários) que utilizam equipamentos médicos nos serviços prestados à comunidade.

\section{Resumos dos Subprojetos:}

Para facilitar a compreensão do leitor, esta seção foi dividida em 4 itens, correspondentes ao resumo de cada subprojeto. De cada subprojeto apresentaram-se os objetivos, a duração, os procedimentos adotados e os resultados. Informações mais pormenorizadas (participantes, formulários e procedimentos desenvolvidos, etc.) sobre o projeto e os subprojetos estão disponíveis no site $\underline{\text { http:// }}$ www.uel.br/projetos/ec $>$.

Subprojeto "Bug do Milênio" (extraído de FRANÇA et al., 2000)

OBJETIVOS: minimizar os efeitos do "bug do milênio" no parque tecnológico instalado no HURNP.

DURAÇÃO: 06/1999 a 11/1999.

\section{PROCEDIMENTOS:}

- organização de um grupo de trabalho com representantes de todos os setores do hospital;

- elaboração de fichas de cadastro para informações dos equipamentos, contendo os seguintes itens: identificação, função, tipo de tecnologia (analógica/digital), controle de data e de hora e sugestões de testes com datas;

- distribuição das fichas para representantes dos setores, que as encaminharam aos responsáveis pela operação dos equipamentos. Depois de preenchidas as fichas, os trabalhos foram divididos em quatro etapas:

- Etapa 1 - análise das informações contidas nas fichas.

- Etapa 2-verificação in loco dos equipamentos e buscas na Internet. 
- Etapa 3 - contato com os fornecedores e fabricantes.

- Etapa 4 - obtenção de certificados dos equipamentos em conformidade com o "bug" e divulgação dos resultados na intranet do HURNP.

\section{Resultados}

Foram cadastrados 545 equipamentos médicos. Deste total, os resultados para as etapas 1, 2 e 3 são apresentados na tabela 1 a seguir. Em cada etapa, foram considerados duvidosos os equipamentos suspeitos de serem afetados pelo "bug do milênio". Deve-se notar também que os equipamentos duvidosos de cada etapa foram os equipamentos analisados na etapa seguinte.

Tabela 1. Resultados das etapas 1, 2 e 3 após análise de sensibilidade dos equipamentos médicos ao "bug do milênio" no HURNP/UEL.

\begin{tabular}{|c|c|c|c}
\hline Etapa & $\begin{array}{c}\text { Equipamentos } \\
\text { sem problemas }\end{array}$ & $\begin{array}{c}\text { Equipamentos } \\
\text { duvidosos }\end{array}$ & $\begin{array}{c}\text { Equipamentos } \\
\text { analisados }\end{array}$ \\
\hline $\mathbf{1}$ & 409 & 136 & 545 \\
$\mathbf{2}$ & 102 & 34 & 136 \\
\hline $\mathbf{3}$ & 32 & 02 & 34 \\
\hline
\end{tabular}

Ao final da Etapa 3, apenas 02 ultra-sons ( $0,37 \%$ do total de 545 equipamentos médicos analisados) precisaram de medidas mais onerosas. Um deles está sendo trocado. Os demais equipamentos que chegaram à Etapa 3 foram atualizados pelos fabricantes. Por isso, com este trabalho, nenhum usuário do HURNP sofreu danos relacionados ao "bug".

\section{Subprojeto "Ar Comprimido"}

OBJETIVOS: redimensionamento e especificação do sistema de ar comprimido do HURNP.

DURAÇÃO: 06/1999 a 02/2003.

\section{PROCEDIMENTOS:}

- levantamento das redes e dos pontos de gases do HURNP (oxigênio, ar comprimido, vácuo e óxido nitroso);

- redimensionamento das instalações e dos equipamentos da central de ar comprimido, de acordo com as estimativas de demanda atual e futura de ar comprimido;

- estudo de opções para solução do problema e escolha da melhor opção (custo $\mathrm{x}$ benefício);

- implementação e supervisão da opção escolhida.

\section{RESULTADOS:}

- estimativa do consumo de ar comprimido no HURNP;

- especificações da nova central de ar comprimido do HURNP;

- sugestão de procedimentos para manutenção preventiva da central de ar do HURNP.

\section{Subprojeto "REFORSUS"}

OBJETIVOS: assessoria técnica e avaliação tecnológica de equipamentos médicos que estão sendo adquiridos pelo HURNP, por meio de um convênio (REFORSUS) com o Ministério da Saúde.

\section{DURAÇÃO: 06/1999 a 02/2003.}

\section{PROCEDIMENTOS:}

- levantamento e especificação dos equipamentos pedidos pelo HURNP;

- aceitação dos equipamentos pedidos (conferência das especificações dos equipamentos com o que foi entregue).

\section{RESULTADOS:}

- formulário desenvolvido no HURNP para recebimento de equipamentos médicos do REFORSUS;

- foram recebidos 122 (93\% dos itens empenhados) equipamentos no valor total de $\mathrm{R} \$ 1,5$ milhão (40\% do total empenhado). 


\section{Subprojeto "Manutenção Preventiva (MP)"}

OBJETIVOS: implementação de um programa de manutenção preventiva nos equipamentos médicos do HURNP.

DURAÇÃO: 06/1999 a 02/2003 (duração contínua).

\section{PROCEDIMENTOS:}

- levantamento dos equipamentos do HURNP;

- priorização dos equipamentos;

- estudar a melhor maneira de fazer a MP;

- especificar esquemáticos, treinamentos, equipamentos de teste;

- gerenciar contratos de MP;

- elaborar cronogramas de manutenção preventiva junto aos setores de enfermagem do HURNP;

- treinar a equipe interna de MP no HURNP.

\section{RESULTADOS:}

- Em 1999/2000 foram especificados e adquiridos alguns equipamentos de teste para o HURNP:

- simulador de $\mathrm{SpO}_{2}$;

- testador de desfibrilador;

- analisador de bombas infusoras;

- analisador de bisturi elétrico;

- analisador de segurança elétrica.

- Em 2001 foi implantado um laboratório para testar esquipamentos na Divisão de Manutenção do HURNP;

- Também em 2001 foram testados até 03 vezes:

- 107 bombas infusoras;

- 60 oxímetros de pulso;

- 22 desfibriladores/cardioversores;

-14 bisturis elétricos.

- Produção Técnica e Científica:

- apostila de matemática básica para alguns servidores de manutenção do HURNP;
- 03 trabalhos apresentados para o Congresso Brasileiro de Engenharia Biomédica de 2002 em São José dos Campos-SP (FELIZARDO et al., 2002; HIRAMA et al., 2002; MAEOKA et al., 2002);

- edição do livro "Manual Hospitalar de Manutenção Preventiva” (RAMÍREZ et al., 2002).

\section{Lições Aprendidas e Contribuições Futuras Esperadas}

Neste trabalho percebeu-se a carência, por parte do HURNP, dos serviços de engenharia clínica. Apesar do trabalho realizado, ainda precisa ser implantada uma gestão efetiva das tecnologias médicas, ou seja, é necessário realmente atuar em todas as etapas do ciclo de vida (aquisição, operação, manutenção e desativação/substituição) dos equipamentos médicos do hospital. Algumas dessas ações poderiam ser traduzidas por atos como:

- participar mais ativamente do processo de especificação e seleção dos equipamentos médicos;

- estender os procedimentos de aceitação utilizados no REFORSUS para os demais equipamentos médicos;

- atuar junto ao setor de patrimônio para implementar uma reestruturação dos sistema de informações ("prontuários") dos equipamentos, promovendo e/ ou auxiliando medidas como a padronização de nomes e colocação ou retirada de informações que permitam uma análise gerencial da divisão de manutenção;

- estender os procedimentos de manutenção preventiva para os demais equipamentos médicos do hospital, por meio do uso de metodologias de priorização e custeio para otimizar a utilização dos recursos humanos e materiais envolvidos;

- realizar uma efetiva gestão dos contratos de manutenção dos equipamentos médicos do hospital, revisando a sua viabilidade, seus custos, os procedimentos e os prazos. 
Para que tudo isso seja colocado em prática, é necessário que o serviço de engenharia clínica no HURNP seja plenamente aceito pelos diversos setores do hospital. Por isso, será necessário incorporálo aos procedimentos administrativos de rotina, pois assim haverá autoridade e legitimidade na atuação do engenheiro clínico. Para tornar isso realidade, propõe-se que estas ações não sejam mais implementadas via projeto de extensão, cuja duração é efêmera, mas sim por institucionalização, mediante convênio entre o curso de Engenharia Elétrica da UEL e o HURNP. Assim, o hospital contaria com recursos humanos para a efetiva gestão de tecnologias médicas e, ao mesmo tempo, serviria como campo de estudo para os docentes e acadêmicos do DEEL na área de Engenharia Clínica. Além disso, este poderia ser um estímulo inicial para a participação do DEEL em projetos de desenvolvimento de equipamentos médicos visando atender as demandas dos profissionais (médicos, enfermeiras e fisioterapeutas) do Centro de Ciências da Saúde (CCS) da UEL.

Enquanto isso não acontece, como a melhoria da qualidade é um processo contínuo, espera-se o prosseguimento dos trabalhos em engenharia clínica no HURNP, mesmo após o término institucional do projeto de extensão citado neste artigo. Para isso pretende-se:

a) Continuar o projeto de manutenção preventiva nos equipamentos médicos do HURNP em conjunto com o pessoal da Divisão de Manutenção (DMPE) do hospital, por meio do:

- recrutamento, seleção e treinamento de estagiários provenientes do curso de Engenharia Elétrica da UEL para desenvolver trabalhos no HURNP;

- desenvolvimento de procedimentos e ensaios técnicos, por meio de estagiários de Iniciação Científica, para avaliar o funcionamento dos diversos equipamentos médicos do HURNP;

- desenvolvimento e aplicação de metodologias de engenharia clínica (otimização de custos, medição de produtividade, priorização de equipamentos e serviços), para facilitar o gerenciamento da manutenção preventiva no HURNP pela Divisão de Manutenção (DMPE / HURNP).

b) Prestar assessoria eventual aos diversos setores do HURNP que envolvam o ciclo de vida de equipamentos médicos por meio da:

- participação da comissão de tecnovigilância da Gerência de Riscos do HURNP para auxiliar a condução das perícias que envolvam a notificação de equipamentos médicos;

- colaboração no processo de recrutamento e seleção de um engenheiro ou tecnólogo para atuar efetivamente como engenheiro clínico do HURNP.

\section{Referências}

ANTUNES, E. et al. Gestão da Tecnologia Biomédica - Tecnovigilância e Engenharia Clínica. Paris: Éditions Scientifiques ACODESS, 2002. $210 \mathrm{p}$.

BETTS, W. F. Cost-effective clinical engineering programs: an expanding role in hospitals. Journal of Clinical Engineering, v.12, n.2, p.119-125, 1987.

BRONZINO, J. D. Management of medical technology: a primer for clinical engineers. Stoneham: Butterworth-Heinemann, 1992. 452p.

CALIL, S. J. Análise do Setor de Saúde no Brasil na área de equipamentos médico-hospitalares. In: NEGRI, B.; DI GIOVANNI, G. (Orgs.). Brasil: Radiografia da Saúde. Campinas: UNICAMP, 2001. p.91-121

CALIL, S.J. Critérios para a Aquisição de Equipamentos Médico-Hospitalares. In: CONGRESSO BRASILEIRO DE ENGENHARIA BIOMÉDICA, 17., 2000, Florianópolis. Anais... Florianópolis, 2000. p.38-45.

CALIL, S. J. The medical equipment acquisition problems in Brazil and most of Latin American countries. Medical Progress Through Technology. v.20, n.1-2, p.111-114, 1994 
CORNIALI, M.C.D.S.; LEITE, H.J.D. Engenharia Clínica e Arquitetura Hospitalar. In: CARVALHO, A.P.A. (Org.). Temas de Arquitetura de Estabelecimentos Assistenciais de Saúde. Salvador: Universidade Federal da Bahia, 2002. p.105-121.

ENDERLE, J. D.; BLANCHARD, S. M.; BRONZINO, J. D. Introduction to Biomedical Engineering. San Diego: Academic Press, 2000. 1062p.

ESPERANÇA, C.G.; VERGARA GALEANO, J.C.; GARCIA OJEDA, R. A Engenharia Clínica e o Processo de Aquisição de Equipamentos Médico-Hospitalares. In: FÓRUM NACIONAL DE CIÊNCIA E TECNOLOGIA EM SAÚDE, 4., 1998, Curitiba. Anais... Curitiba, 1998. p.143-144.

FELIZARDO, K.R.; ITANO, M.E.; RAMÍREZ, E.F.F. Controle de Qualidade de Oxímetros de Pulso em Hospitais. XVIII CONGRESSO BRASILEIRO DEENGENHARIA BIOMÉDICA,18., São José dos Campos, 2002. Anais... São José dos Campos: Univap, 2002. v.2/5, p.41-45.

FRANÇA, M.B.M.; RAMÍREZ, E.F.F.; GOMI, J. "Bug" 2000 nos Equipamentos Médicos do HURNP. Londrina. In: ENCONTRO REGIONAL DE INICIAÇÃO CIENTÍFICA DA UNOPAR, 1., 1999, Londrina. Anais... Londrina, 1999.

GOMES, A.C.; GOMES, A.C. Incorporação de Novas Tecnologias nos Sistemas de Atenção à Saúde. do IV FÓRUM NACIONAL DE CIÊNCIA E TECNOLOGIA EM SAÚDE, 4., Curitiba, 1998. Anais... Curitiba, 1998. p.123-124.

HAWKINS, F.G. A Review of Issues in Hospital Technology Acquisition. Journal of Clinical Engineering, v.17, n.1, p.35-41, 1992

HIRAMA, R.T. et al. Método para Inspeção de Bombas Infusoras. In: CONGRESSO BRASILEIRO DE ENGENHARIA BIOMÉDICA, 18., 2002, São José dos Campos. Anais... São José dos Campos: Univap, 2002. v.2/5, p.35-40.
LOPES, A.F. Avaliação do Processo de Aquisição de Equipamentos Médicos. 1993. Dissertação (Mestrado em Engenharia) - Coordenação dos Programas de Pós-Graduação de Engenharia da Universidade Federal do Rio de Janeiro, Rio de Janeiro, 1993.

MAEOKA, G.K. et al. Metodologia para Avaliação de Desfibriladores e Cardioversores. In: CONGRESSO BRASILEIRO DE ENGENHARIA BIOMÉDICA, 18., 2002, São José dos Campos. Anais... São José dos Campos: Univap, 2002. v.2/5, p.46-50.

NETTO, E.J. et al. A Engenharia Hospitalar no Estado do Paraná. Revista Brasileira de Engenharia Caderno de Engenharia Biomédica, v.6, n.2, p.235239, 1989.

PANOUSIS S.G. et al. A study on the current situation in the biomedical technology and clinical engineering sector in Bulgaria - advances, trends and needs. Journal of Clinical Engineering, v.22, n.6, p.391-399, 1997.

RAMÍREZ, E.F.F.; CALIL, S.J. Engenharia Clínica: Parte I - Origens (1942-1996). Semina: Ci. Exatas/Tecnológicas. Londrina, v.21, n.4, p.27-33, dez. 2000.

RAMÍREZ, E.F.F.; CALDAS, E.C.; SANTOS JR., P.R. Manual Hospitalar de Manutenção Preventiva. Londrina: EDUEL, 2002. 180p.

SOUZA, M.C. D.; SILVEIRA, M.; SILVA, J.F. Análise do Processo de Aquisição de Equipamento Odonto-Médico-Hospitalar para a SESAB - Estudo de Caso: Projeto Reforsus. In: CONGRESSO BRASILEIRO DE ENGENHARIA BIOMÉDICA, 17., 2000, Florianópolis. Anais... 2000. p.418-422. 